of cysts. The encephaloid masses were chiefly situated in the posterior and lower parts of the tumour; they had a reddishwhite, vascular appearance, and a soft consistence; they varied in size from that of an orange to that of a walnut, and some of them were beginning to break down into a semi-liquid, grumous matter. The hemorrhagic masses were composed of soft clotted blood, mixed with disintegrated cancerous matter in the upper part there was a mass of pure clotted blood as large as the fist. The cysts were some eight or ten in number; the two largest had been punctured during life, and were now partially collapsed and half filled with a turbid reddish fluid, which, under the microscope, had the characters of imperfect pus. The unbroken cysts varied from the size of a marble to that of a small apple; some contained clear, yellow, diffuent gerum, and others jelly-like material.

$$
\text { FIG. } 2 .
$$

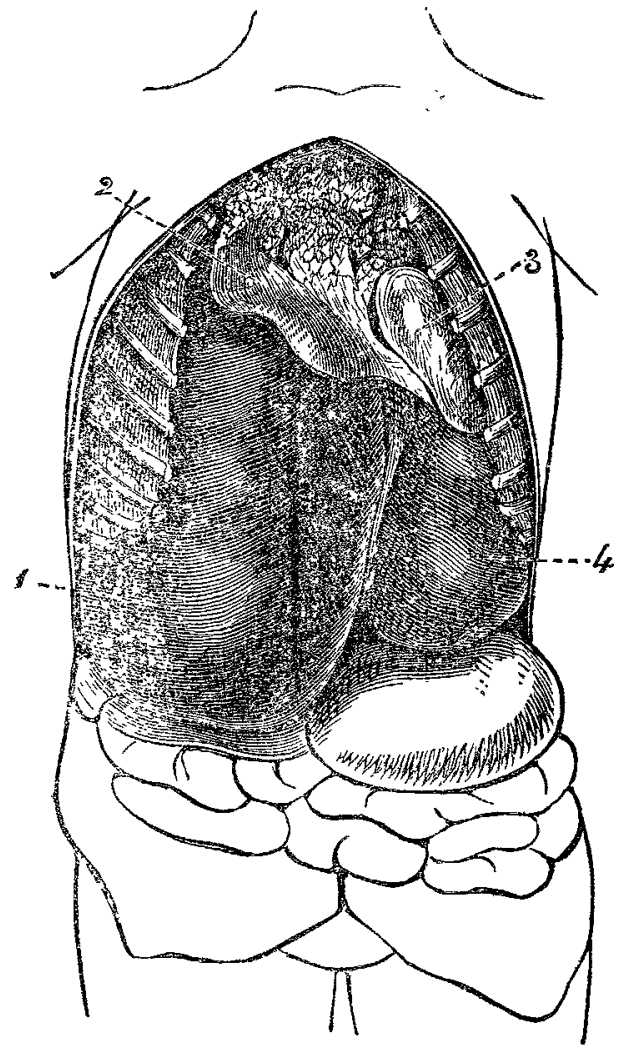

Showing the relations of the tumour to the surrounding iscera. 1. Tumour. 2. Remnant of right lung. 3. Peri cardium. 4. Left lobe of liver.

The tumour measured 13 in. in length and $12 \frac{1}{2}$ in. in breadth, including the left lobe; its greatest thickness was $5 \frac{1}{2}$ in. The weight of the entire mass was $10 \mathrm{lbs}$.; the tumour itself weighed about $S \mathrm{lbs}$. The left lobe of the liver contained no cancerous masses, and no secondary cancerous deposits existed in any of the other organs or tissues of the body.

The present case, while differing wholly from the ordinary types of hepatic cancers, resembled, structurally, the common form of fungus hæmatodes of the testicle. It resembled still more closely the remarkable cancerous masses found in the kidneys of young children. In these latter cases enormous masses are sometimes found, weighing ten, twenty, and even thirty pounds, composed, like the tumour in the case just described, of encephaloid masses, masses of extravasated blood, and serous cysts.

ON

\section{THE TREATMENT OF FRACTURES OF THE} LOWER JAW.

BY H. O. THOMAS, M.R.C.S.

I DESIRE, through the medium of THE LANCET, to request those of my professional brethren who have the opportunity to test the usefulness of a plan I have practised the last four years in treating fractures of the lower jaw. It would best illustrate my treatment to describe one or two cases.
T. S - whilst engaged in a street row, received a blow on the lower jaw, on the 5th of April, 1866. I examined the jaw the following morning, and found a fracture at the symphysis, with great mobility of the fracture. Having requested an assistant to steady the head, whilst another drew down and everted the lower lip, a fine drill was passed (at the point of reflection of the mucous membrane) through the jaw, about a quarter of an inch on cither sicle of the fracture, using an ordinary Archimedean drill, one-sixteenth of an inch in size. I then passed through a silver wire, about the strength of that used by whip-makers. Having secured the ends in front of the jaw, they were then drawn tight and twisted until the fracture was firmly fixed. On the fifth day it became a little slack, and was tightened by an extra twist, which required to be repeated every three or four days. In twenty days there was but slight motion at the fracture, and on the twenty-eighth it was quite firm and united. The patient from the first day expressed himself as able to use the jaw, and was urgent to be allowed to do so, which was not permitted.

Thomas B-, a ship-carpenter, was struck by a piece of timber on the face, which threw him from the stage on which he worked, and he fell a depth of seventeen feet. On examining him an hour after the accident, there was found a fracture of the lower jaw on the right side, at the situation of the first and second molar teeth, which had been knocked out by the force of the blow. There was great mobility of the fractured part, more than I recollect seeing before in fractures in that situation. The remaining teeth were firmly in situ. Having had the inside of the mouth well exposed by drawing aside the cheek, my assistant kept the third molar tooth steady with a piece of wood directed across the mouth from the left side whilst a hole was drilled across the tooth from its front to its inner surface, this tooth holding firm in the posterior portion of the fracture. A strong silver wire was then passed through the hole and brought forward, passing it between the bicuspid and canine teeth; the ends were then drawn tight and twisted, making the fracture firm. The wire was tightened every four days, and in three weeks there was moderate union; in four weeks it was sufficiently secure to allow the wire to be removed and the jaw used. This was the only treatment in the shape of mechanical appliance. An opiate was given every other night. This patient was also with difficulty restrained from using his jaw for masticating.

A case also came under my care four years ago, which occurred in a patient whilst engaged in "docking a ship" when he was struck on the jaw by a "capstan-bar," and a portion of the lower jaw, corresponding to the two middle incisor teeth, was detached and afterwards removed. The same treatment was followed as described in the first case, resulting in firm union in ten weeks.

Many cases of fractured jaw do well with comparatively simple treatment; and in cases where there has been great violence and much mobility of the fractured bones, the above practice of securing the body of the bone by means of wires in the way described, I believe to be peculiarly advantageous, to the exclusion, with few exceptions, of all other apparatus, and applicable to almost any form of fracture of the body of the jaw.

The passage of a fine drill in the situation described in the above cases does not appear to have endangered the teeth, care being taken to strike, if possible, the interspaces of their roots. The body of the tooth which was drilled in the second case was attended with but little discomfort to the patient and which the skill of the dentist will probably make good again.

Nelson-street, Liverpool, Jan. 1867.

\section{A NEW INSTRUMENT FOR INJECTING CANCEROUS TUMOURS OF THE UTERUS AND RECTUM.}

\section{By T. J A T TON,}

CONSULTING SURGEON TO TEE MARYLEBONR INFTRMARY.

THE philosophic suggestion of Dr. Broadbent for the treatment of cancer is now being put to the test of experience by several eminent members of the profession. The time at present, however, has been too limited, and the observations too few, to admit of any positive deductions as to the attainment of the earnestly desired result-a cure for cancer. 